\title{
BMJ Open Participatory approaches in the context of research into workplace health promotion to improve physical activity levels and reduce sedentary behaviour among office-based workers: protocol for a scoping review
}

Aidan John Buffey (D) ,1,2 Brian Carson,, ${ }^{1,2}$ Alan Donnelly, ${ }^{1,2}$ Jon Salsberg (D) 3,4

To cite: Buffey AJ, Carson B, Donnelly $A$, et al. Participatory approaches in the context of research into workplace health promotion to improve physical activity levels and reduce sedentary behaviour among office-based workers: protocol for a scoping review. BMJ Open 2021;11:e054402. doi:10.1136/ bmjopen-2021-054402

- Prepublication history and additional supplemental material for this paper are available online. To view these files, please visit the journal online (http://dx.doi.org/10.1136/ bmjopen-2021-054402)

Received 10 June 2021 Accepted 25 November 2021

Check for updates

(C) Author(s) (or their employer(s)) 2021. Re-use permitted under CC BY-NC. No commercial re-use. See rights and permissions. Published by BMJ.

For numbered affiliations see end of article.

Correspondence to Mr Aidan John Buffey; Aidan.Buffey@ul.ie

\section{ABSTRACT}

Introduction Physical activity (PA) workplace health promotion (WHP) interventions have traditionally used a top-down research approach where end-users are considered as passive subjects. Whereas participatory research (PR) involves the end-users within the research process utilising a bottom-up approach which allows the integration of the researcher's expertise and the end-users lived experiences, which has been shown to aid in the acceptability and relevance of the research. This protocol describes a scoping review which will explore, identify and map PR techniques and their impact when used in officebased WHP interventions designed to improve PA and reduce sedentary behaviour levels.

Methods and analysis This scoping review will follow the guidelines and framework proposed by Levac et al and Preferred Reporting Items for Systematic reviews and Meta-Analyses extension for Scoping Reviews. Articles will be retrieved via five databases: Web of Science, PubMed, Scopus, Google Scholar and OpenGrey, searching from 1 January 1995, to the date of search in 2022. A search strategy was piloted, to identify appropriate keywords and MeSH terms. Two independent reviewers will screen retrieved articles based on our inclusion and exclusion criteria by title and abstract first, followed by the full text. Our key inclusion criteria relating to our population, intervention, comparison, outcome framework will be to include studies which focused on office-based working adults and that the PA WHP took a PR approach. Articles written in non-English languages will be excluded. Any discrepancies will be discussed until a consensus is reached. Data will be extracted, charted and summarised via a narrative synthesis and qualitative thematic analyses.

Ethics and dissemination Ethical approval was not required for this scoping review. The completed scoping review findings will be disseminated in a peer-reviewed journal which has a research scope that encompasses PR and WHP. The findings will be presented at appropriate academic conferences and to project partners to inform the design of a WHP intervention.
Strengths and limitations of this study

- The proposed scoping review will explore and map the current participatory research techniques and approaches taken when completing an office-based workplace health promotion intervention designed to increase physical activity and reduce sedentary behaviour.

- The scoping review by nature and the search strategy proposed will allow for a wide breadth of literature to be explored.

- This scoping review will exclude studies written in non-English languages, and therefore, could decrease the number of eligible articles otherwise.

\section{INTRODUCTION}

Within occupational health research, prolonged occupational sitting is a topic of increased debate and growing research. ${ }^{1}$ Changes in the workplace environment have been associated with significant reductions in the demand of physical activity (PA) and the increased use of computers has shown an increased prevalence of prolonged sitting in many workplaces, especially office-based workplaces. ${ }^{23}$ Sitting is classified as a sedentary behaviour (SB) which is a term used to classify low levels of energy expenditure when sitting or in a reclined posture and lying. ${ }^{3}$ The workplace environment and organisational culture can often facilitate and promote prolonged SB. ${ }^{2}{ }^{4}$ Two previous studies objectively measured sedentary time, using accelerometers, in office workers. ${ }^{5}{ }^{6}$ They found office workers were sedentary for a mean of $75.8 \%(95 \% \text { CI } 74.5 \% \text { to } 77.1 \%)^{6}$ and $81.8 \%$ $(438.8 \pm 51.5 \mathrm{~min})^{5}$ of their working hours.

Previous workplace health promotion (WHP) interventions have taken a traditional 
top-down research-driven approach where the end-users are considered as passive subjects. ${ }^{7-9}$ In this review, an end-user is defined as an individual working in an office environment or communities such as the organisations workforce. Whereas participatory research (PR) is an approach to research that involves the target end-user and relevant stake holders within the research process to promote and establish a sense of ownership which helps to increase the relevance of the research. ${ }^{7}$ This sense of ownership is seen in health promotion (HP) research which at its philosophical core focuses on empowerment and community participation. ${ }^{10} \mathrm{PR}$ allows the integration of the researchers' expertise and the target populations lived experiences which is a significant strength of the PR approach to research and has been shown to aid the acceptability of the research when implemented if conducted well. ${ }^{11}$ An early description of HP by the WHO establishes HP as a method of empowering individuals and communities to take control over their health and its determinants. ${ }^{12}$

Many WHP interventions have been conducted and targeted different aspects of either the workplace environment, workhours schedule, transport to and from work or targeted behavioural changes to increase PA and reduce $\mathrm{SB}$, either looking at the different behaviours collectively or individually. Some examples are (A) taking the stairs, in place of an elevator, (B) encourage achieving the recommended moderate-to-vigorous PA guidelines, (C) active emails and or walking meetings, (D) active transport (walking or cycling to and from work), (E) height adjustable desks, treadmill desks and or cycling desks, (F) pedometers/accelerometers that measure PA and (G) breaking SB. ${ }^{13513}$ These interventions, however, are typically administered at an individual level with a traditional top-down research approach. Previous WHP interventions that did not implement a participative approach to the intervention when targeting behaviour change have been shown to be weaker in design. ${ }^{14}$ While workplace interventions that employed a participatory approach accounting for factors such as individual, interpersonal, organisational, workplace community, company policy and workplace environment and the interaction between these factors are considered multilevel interventions. ${ }^{1}$ It has been proposed that multilevel interventions are typically more effective than individual level interventions when aiming to increase moderate to vigorous PA. ${ }^{1}$ Multilevel interventions conducted with a PR approach have been shown to be beneficial in numerous ways.

Creating a PA WHP intervention which is sustainable after the completion of the study should be a research priority and maintaining end-user adherence during the intervention can be difficult, with high rates of attrition shown in previous WHP studies. ${ }^{13}$ For example, participants who are highly sedentary prior to an intervention are likely to return to their previous $\mathrm{SB}$, due to increasing work pressures. ${ }^{2}$ Therefore, studies using a PR approach which fosters a motivational component to the study for the participant may be beneficial. ${ }^{2}$ This inclusivity with the end-users, with the aim of collaboration, education and community action promotes active involvement within the research process. ${ }^{71516}$

Previous research investigating work site health has taken traditional research approaches, such as; in-person interventions, printed materials and information talks. ${ }^{17}$ Malik et $a l^{13}$ completed a systematic review of WHP interventions designed to promote $\mathrm{PA}$ and evaluated the impact they had on participants' PA levels. They found evidence to suggest WHP interventions can be effective and showed positive outcomes for some of the included studies, however, the overall results were inconclusive and called for more research into the elements of WHP interventions that are likely to increase efficacy and adoption within the workplace. ${ }^{13}$ This scoping review will extend on Malik $e a^{13}$ as it will investigate WHP interventions that have taken a PR approach within their study. Previous literature has shown that a PR approach can increase the efficacy and lead to successful implementation and greater adherence within HP studies. ${ }^{11} 1415$ Thus, this scoping review will provide evidence on the use of PR in WHP studies that may lead to greater adoption of PR and success of WHP interventions.

A PR approach can be taken in a variety of forms and at different stages of an intervention (eg, planning, conducting, evaluating, reporting and disseminating). Along with varying methodologies, which can impact the level of effectiveness seen in a WHP intervention. When a participatory approach is not taken, previous research has shown that the WHP study may lead to an intervention approach/concept or format that is inappropriate. ${ }^{18}$ Previous literature has examined the benefits and effectiveness of WHP interventions. However, to the authors' knowledge, the use of PR within WHP intervention has not been synthesised. Therefore, the authors aimed to examine how PR is being incorporated within WHP research, to identify the current available evidence, key approaches and methods, and the scope of reported impacts of PR. Providing an overview and identifying key characteristics of the current research that has utilised PR within the WHP intervention aimed to increase PA and reduce SB. Due to the broad nature of the scoping review, we developed key research objectives, these being:

1. Identify and map previous literature where officebased adults have been involved in PR studies and how their involvement shaped the design of the WHP intervention.

2. Identify and discuss the methods implemented in the WHP studies that took a PR approach.

3. Discuss the evaluation and outcomes measured in the WHP articles included in the scoping review that took a PR approach.

A scoping review was, therefore, identified as the most appropriate methodological approach to investigate the use of PR within WHP interventions and to map and explore all available evidence and identify and analyse any knowledge gaps. ${ }^{19}$ 


\section{METHODS AND ANALYSIS}

\section{Protocol}

The methodology for this scoping review will follow the guidelines and framework published by Levac et $a t^{20}$ who extended and advance the methodology of scoping reviews from previous researchers framework, Arksey and O'Malley. ${ }^{21}$ The extended framework consists of six stages: (1) Identifying the research question ; (2) Identifying relevant studies; (3) Study selection; (4) Charting the data; (5) Collating, summarising and reporting the results and (6) an optional stage, Consultation. ${ }^{20}$ The reporting will follow the guidelines and framework developed from the Preferred Reporting Items for Systematic Reviews and Meta-Analyses extension for Scoping Reviews (PRISMA-ScR) ${ }^{22}$ The PRISMA-ScR consists of a 22-item checklist (Found: http://www.prisma-statement.org/ documents/PRISMA-ScR-Fillable-Checklist_11Sept2019. pdf).

\section{Stage 1: identifying the research question}

To guide the scope of inquiry and purpose of the scoping review when identifying our research question, we considered the intended outcome. The intended outcome for this review was to see the extent of available literature and provide researchers and stakeholders literature on PR approaches and techniques within office-based PA WHP studies and how PR may strengthen or put limitations on WHP studies. Followed by providing recommendations when incorporating PR in PA WHP studies. To clarify the focus of the study and identify the research question we considered the concept, target population and health outcomes of interest and used the PICO framework. Our $(\mathrm{P})$, population, is office-based working adults, (I), intervention, is PA WHP interventions that used a PR approach, (C), comparison, we do not wish to compare interventions or treatments (this is typical in some PICO analysis frameworks, where a comparison is not always present). (O), outcome, PA and SB levels.

Our intended outcome and purpose, along with the PICO framework helped identify and formulate our research question:

'How have previous PA WHP studies, investigating office-based adults, incorporated PR and the end-user within their studies and to what benefit or detriment is reported?'

\section{Stage 2: identifying relevant studies}

A systematic search spanning five electronic databases, which includes Web of Science, PubMed, Scopus, Google Scholar and OpenGrey will be completed between the years of 1 January 1995 to the date of search in 2022. When searching Google Scholar, the first 100 results retrieved will be screened for eligibility. Articles will be screened for eligibility relating to our inclusion and exclusion criteria, including the exclusion of non-English written articles (see table 1).

To best capture the breadth of literature that we were hoping to retrieve that fit our eligibility criteria (see table 1), we piloted preliminary searches and referred to previous review articles search terms in the research area of WHP interventions. ${ }^{13}$ After performing preliminary searches of the Web of Science database and identifying keywords from article titles and abstracts, we identified the Medical Subject Headings (MeSH) terms of these keywords using PubMed. These keywords and MeSH terms were then used across all included databases and adapted where needed across the databases. A complete search strategy for each database searched is included in the online supplemental file 1 .

\section{Stage 3: study selection}

Two independent reviewers (AJB and CL) will complete the search strategy, independently and in duplicate. Duplicated articles will be identified and removed via Endnote Online, with the retrieved articles then being screened using Microsoft Excel (Microsoft Excel, 2011). The title and abstract of the retrieved articles will then be screened for eligibility, followed by the full text. The retrieved articles will be annotated 'Yes' or 'No' for inclusion, followed by the researcher's reason in the subsequent column within Microsoft Excel (Microsoft Excel, 2011). Any disagreement between the two reviewers will be resolved through discussion based on the inclusion and exclusion criteria between the two reviewers. If a decision on inclusion cannot be resolved between the two reviewers, arbitration by a third reviewer (JS) will take place, until a consensus is reached. Grey literature will be screened from OpenGrey and Google Scholar (theses/ dissertations, conference papers, research/government reports, ongoing research, editorials and textbooks) in the same process as the articles retrieved from Web of Science, PubMed, Google Scholar and Scopus. The reference lists of the included studies will be screened, and where any relevant literature is found, these will be included. The PRISMA flow diagram template will be published alongside the scoping review to illustrate the search strategy screening process, providing the number of sources screened, with reasons for exclusion and the final number of included studies. ${ }^{22}$

Table 1 Illustrates the eligibility criteria, with the inclusion and exclusion criteria and rationale statements.

\section{Stage 4: charting the data}

Data from the retained studies will be charted independently by one reviewer with a random selection of $10 \%$ of the included studies being independently duplicated by a second reviewer to confirm the data charting process. Any discrepancies will be discussed until a consensus is reached, arbitrated when needed by a third reviewer. If discrepancies are identified, the charting process for all the included studies will be rechecked by both reviewers. Data will be extracted from the included studies and charted into a Microsoft Excel sheet (Microsoft Excel, 2011) table. The data extracted will be charted into the Microsoft Excel sheet following the headings 
Table 1 Eligibility criteria

\begin{tabular}{lll}
\hline Characteristics & Inclusion & Exclusion \\
\hline Population & $\begin{array}{l}\text { Humans. } \\
\text { Working adults. } \\
\text { Office environment. }\end{array}$ & Home-based 'office' \\
& workers. \\
& \\
& \\
& \\
\end{tabular}

Language English language.

\section{Rationale}

The focus of this scoping review is to investigate PR in WHP studies in office-based participants/ workplaces.

Children, teenagers and retired adults would not fit our eligibility criteria of 'working adults'.

Non-office workers and home-based workers may have different 'health' needs relating to the working environment.

The reviewers only speak English and feasibility considerations (eg, limited resources) prevent the use of translation services.

$\begin{array}{ll}\begin{array}{l}\text { Years } \\ \text { considered }\end{array} & \text { (not yet complete), } 2022\end{array}$

considered (not yet complete), 2022

A wide time period to capture all relevant WHP research.

PR became more prominent in HP studies in 1995 and therefore implemented into research practices following this year.
Study focus Articles investigating WHP in office-based workplaces that implemented participatory health research techniques. Includes a PA aspect to the intervention study, for example, increasing PA or decreasing SB.
Studies must be conducted The focus of the overall research question of this within the workplace with scoping review, is specific to PR in WHP research in the aim of improving health. office-based workplaces.

Not based in the community Other work-based environments may carry different or home.

The health promotion intervention was measuring/ based environment.

targeting psychological or work-performance improvements.

Any other literature that is not listed in the inclusion criteria, such as websites. health-associated risks, priorities or safety concerns which would not be comparable to that of an officeIncluding outcome measures will allow for an evaluation of the included studies and a discussion on the effectiveness of taking a PR approach in those WHP studies.

The aim of this scoping review is to capture a wide breadth of literature so including grey literature ensures a more complete search and minimises

$\begin{array}{ll}\text { Publication } & \text { Published peer-reviewed } \\ \text { status } & \text { journal articles and relevant } \\ & \text { grey literature. Relevant grey } \\ & \text { literature is defined within this } \\ & \text { scoping review as: theses/ } \\ \text { dissertations, conference papers, } & \text { research/government reports, } \\ & \text { ongoing research, editorials and } \\ & \text { textbooks. }\end{array}$
publication bias. shown below and filled in with information answering the associated questions (see table 2).

\section{Stage 5: collating, summarising and reporting the results}

Based on Levac et $a l^{20}$ and their recommendations and advancements from Arksey and O'Malley's ${ }^{21}$ framework, we have split stage 5 into three distinct steps.

\section{Step 1: analysis}

Following data extraction and charting we will provide a narrative synthesis of the included studies, descriptively summarising the data that has been charted. We will not critically appraise the data; we will look to aggregate the findings of the included studies allowing us to summarise the results descriptively and numerically. Our decision to not critically appraise the included studies is a standard approach when conducting scoping reviews. ${ }^{19} 21$ In line with the Levac et $a l^{20}$ framework, we will conduct qualitative thematic analysis. Our thematic analysis will follow the guidance of Braun and Clarke 23 utilising their developed '15-point checklist of criteria for good thematic analysis'. Where data has been extracted relating to changes in $\mathrm{PA}$ and $\mathrm{SB}$, whether negative, positive or neutral, we will present these findings descriptively. We will discuss the methods and characteristics of the studies related to the change in $\mathrm{PA}$ and $\mathrm{SB}$.

\section{Step 2: reporting}

The narrative synthesis of results and findings from the qualitative thematic analysis will be reported and organised into thematic categories. Some example categories we intend to report on would be; methodological design, end-user role and level of involvement; impact on PA and SB levels; PR benefits and PR limitations within PA WHP studies. Reporting on these categories will address the overall purpose, research question and objectives of the scoping review. 
Table 2 Data charting headings and associated questions used to retrieve information from the included articles

\begin{tabular}{|c|c|}
\hline Charted data & Associated questions \\
\hline First author & What is the name of the first author? \\
\hline Title of journal article & What is the title of the published article? \\
\hline Origin & Where was the study conducted? \\
\hline Population & What are the descriptive characteristics of the studies participant sample group? \\
\hline $\begin{array}{l}\text { Study purpose and } \\
\text { aims }\end{array}$ & $\begin{array}{l}\text { What was the purpose of the study? } \\
\text { What were the aims/objectives of the study? }\end{array}$ \\
\hline Study procedures & $\begin{array}{l}\text { What was the duration of the study? } \\
\text { When and how were measures taken (baseline, outcomes, follow-up)? }\end{array}$ \\
\hline PR techniques & $\begin{array}{l}\text { What PR techniques are mentioned, detailed, and implemented within the methodology of the study? } \\
\text { How and where within the study were the PR techniques implemented? }\end{array}$ \\
\hline Intervention focus & $\begin{array}{l}\text { Was there an intervention? What did the intervention focus on/measure? } \\
\text { What was the PA intervention? Methods and details. }\end{array}$ \\
\hline Data collection & How was data collected? Note down recorded findings. \\
\hline Study outcomes & What were the study outcomes? Note down the reported study outcomes. \\
\hline Data analysis & How was data analysed? Was a PR approach taken concerning data analysis or data checking? \\
\hline $\begin{array}{l}\text { Evaluation of PR } \\
\text { techniques }\end{array}$ & $\begin{array}{l}\text { Did the WHP intervention evaluate any of the PR techniques implemented? If so, how? Note down their } \\
\text { self-evaluation. } \\
\text { Following Jagosh et al, }{ }^{25} \text { self-reported PR will be assessed by asking of the article; 'Does the full-text } \\
\text { paper indicate that participation occurred in the following three areas: (A) Partners were involved in } \\
\text { identifying or setting the research questions? (B) Partners were involved in setting the methodology } \\
\text { or collecting data or analysing the data? (C) Partners were involved in uptake or dissemination of the } \\
\text { research findings (this requirement will be loosely applied because publication often predates uptake)? }\end{array}$ \\
\hline Notes & $\begin{array}{l}\text { Any information that may be useful that does not fit into the above questions asked of the retrieved } \\
\text { studies? }\end{array}$ \\
\hline
\end{tabular}

PA, physical activity; PR, participatory research; WHP, workplace health promotion.

Step 3: implications for future research, practice and policy

By understanding how PR has been implemented and evaluated within PA WHP interventions, we expect the findings from this scoping review will inform future research questions; provide a base of current evidence, indicate the key methods when implementing PR within PA WHP interventions and the scope of reported impacts of PR. We plan to provide recommendations on the implementation of PR techniques in PA WHP studies and the involvement of endusers and their level of involvement within the different stages of planning and completing a PA WHP study.

\section{Stage 6: consultation with knowledge users}

This scoping review will be presented during the planning stage of a clustered randomised WHP study. As part of the formation of the study, this scoping review will be used to inform and guide stakeholders (eg, managers and/or business owners) who are involved in the project when planning which and how the PR approach will be taken, and the role and the level of involvement end-users will take.

\section{Patient and public involvement}

End-users and stakeholders were not involved in the development of this scoping review protocol, nor will they be in the completion of the scoping review. While we intend to discuss the findings with project partners and stakeholders who will be involved in later stages of the overall project, such as the WHP study, we want to limit the time burden and commitment to end-users at this stage. 


\section{ETHICS AND DISSEMINATION}

\section{Ethics}

Ethical approval was/is not required or obtained for this scoping review protocol or the scoping review.

\section{Dissemination}

We plan to disseminate the findings of the completed scoping review through publication, in a peer-reviewed journal that incorporates PR and HP within the scope of the journal as well as presenting the findings at appropriate academic conferences. The findings of the completed scoping review will be presented to project partners and stakeholders during the planning of a WHP intervention.

\section{Author affiliations}

${ }^{1}$ Department of Physical Education and Sport Science, University of Limerick Faculty of Education and Health Sciences, Limerick, Ireland

${ }^{2}$ Physical Activity for Health Research Cluster, Health Research Institute (HRI), University of Limerick, Limerick, Ireland

${ }^{3}$ Public and Patient Involvement Research Unit, School of Medicine, University of Limerick, Limerick, Ireland

${ }^{4}$ Public and Patient Involvement Research Unit, Health Research Institute (HRI), University of Limerick, Limerick, Ireland

\section{Twitter Aidan John Buffey @AidanBuffey and Jon Salsberg @jsalsb}

Acknowledgements CL refers to Miss Christina Langley (Manchester Metropolitan University), who will contribute to the scoping review in the search strategy, data charting and editing of the scoping review. CL has not contributed to the planning of the scoping review protocol or manuscript.

Contributors All authors made substantial intellectual contributions to the development of this protocol. AJB and JS conceptualised the review approach and AJB drafted the manuscript. $B C, A D$ and JS contributed to the conceptualisation, writing and editing of the protocol.

Funding This research was supported by the School of Medicine (Grant Number: $\mathrm{N} / \mathrm{A}$ ), University of Limerick and a University of Limerick, Health Research Institute Studentship.

Competing interests None declared.

Patient consent for publication Not applicable.

Provenance and peer review Not commissioned; externally peer reviewed.

Supplemental material This content has been supplied by the author(s). It has not been vetted by BMJ Publishing Group Limited (BMJ) and may not have been peer-reviewed. Any opinions or recommendations discussed are solely those of the author(s) and are not endorsed by BMJ. BMJ disclaims all liability and responsibility arising from any reliance placed on the content. Where the content includes any translated material, BMJ does not warrant the accuracy and reliability of the translations (including but not limited to local regulations, clinical guidelines, terminology, drug names and drug dosages), and is not responsible for any error and/or omissions arising from translation and adaptation or otherwise.

Open access This is an open access article distributed in accordance with the Creative Commons Attribution Non Commercial (CC BY-NC 4.0) license, which permits others to distribute, remix, adapt, build upon this work non-commercially, and license their derivative works on different terms, provided the original work is properly cited, appropriate credit is given, any changes made indicated, and the use is non-commercial. See: http://creativecommons.org/licenses/by-nc/4.0/.

\section{ORCID iDs}

Aidan John Buffey http://orcid.org/0000-0002-1940-1483

Jon Salsberg http://orcid.org/0000-0003-2010-3691
REFERENCES

1 Plotnikoff R, Karunamuni N. Reducing sitting time: the new workplace health priority. Arch Environ Occup Health 2012;67:125-7.

2 Brown DK, Barton JL, Pretty J, et al. Walks4Work: assessing the role of the natural environment in a workplace physical activity intervention. Scand J Work Environ Health 2014;40:390-9.

3 Owen N, Healy GN, Matthews CE, et al. Too much sitting: the population health science of sedentary behavior. Exerc Sport Sci Rev 2010;38:105-13.

4 Marshall AL. Challenges and opportunities for promoting physical activity in the workplace. J Sci Med Sport 2004;7:60-6.

5 Parry S, Straker L. The contribution of office work to sedentary behaviour associated risk. BMC Public Health 2013;13:296.

6 Thorp AA, Healy GN, Winkler E, et al. Prolonged sedentary time and physical activity in workplace and non-work contexts: a cross-sectional study of office, customer service and call centre employees. Int J Behav Nutr Phys Act 2012;9:1-9.

7 Macaulay AC, Commanda LE, Freeman WL, et al. Participatory research maximises community and lay involvement. BMJ 1999;319:774-8.

8 Potvin L, Cargo M, McComber AM, et al. Implementing participatory intervention and research in communities: lessons from the Kahnawake schools diabetes prevention project in Canada. Soc Sci Med 2003;56:1295-305.

9 Jagosh J, Macaulay AC, Pluye P, et al. Uncovering the benefits of participatory research: implications of a realist review for health research and practice. Milbank Q 2012;90:311-46.

10 Robertson A, Minkler M. New health promotion movement: a critical examination. Health Educ Q 1994;21:295-312.

11 Cargo M, Mercer SL. The value and challenges of participatory research: strengthening its practice. Annu Rev Public Health 2008;29:325-50.

12 World Health Organisation. Discussion document on the concept and principles of health promotion. Copenhagen: European Office of the World Health Organization, 1984.

13 Malik SH, Blake H, Suggs LS. A systematic review of workplace health promotion interventions for increasing physical activity. $\mathrm{Br} \mathrm{J}$ Health Psychol 2014;19:149-80.

14 Parry S, Straker L, Gilson ND, et al. Participatory workplace interventions can reduce sedentary time for office workers--a randomised controlled trial. PLoS One 2013;8:e78957.

15 De las Nueces D, Hacker K, DiGirolamo A, et al. A systematic review of community-based participatory research to enhance clinical trials in racial and ethnic minority groups. Health Serv Res 2012;47:1363-86.

16 Shimmin C, Wittmeier KDM, Lavoie JG, et al. Moving towards a more inclusive patient and public involvement in health research paradigm: the incorporation of a trauma-informed intersectional analysis. BMC Health Serv Res 2017;17:539.

17 Cook RF, Billings DW, Hersch RK, et al. A field test of a web-based workplace health promotion program to improve dietary practices, reduce stress, and increase physical activity: randomized controlled trial. J Med Internet Res 2007;9:e17.

18 Rojatz D, Merchant A, Nitsch M. Factors influencing workplace health promotion intervention: a qualitative systematic review. Health Promot Int 2017;32:831-9.

19 Munn Z, Peters MDJ, Stern C, et al. Systematic review or scoping review? Guidance for authors when choosing between a systematic or scoping review approach. BMC Med Res Methodol 2018;18:143.

20 Levac D, Colquhoun H, O'Brien KK. Scoping studies: advancing the methodology. Implement Sci 2010;5:69.

21 Arksey H, O'Malley L. Scoping studies: towards a methodological framework. Int J Soc Res Methodol 2005;8:19-32.

22 Tricco AC, Lillie E, Zarin W, et al. PRISMA extension for scoping reviews (PRISMA-ScR): checklist and explanation. Ann Intern Med 2018;169:467-73.

23 Braun V, Clarke V. Successful qualitative research: a practical guide for beginners. New York: Sage, 2013.

24 Braun V, Clarke V. Using thematic analysis in psychology. Qual Res Psychol 2006;3:77-101.

25 Jagosh J, Pluye P, Macaulay AC, et al. Assessing the outcomes of participatory research: protocol for identifying, selecting, appraising and synthesizing the literature for realist review. Implement Sci 2011;6:24.

26 Arnstein SR. A ladder of citizen participation. J Am Inst Plann 1969;35:216-24. 